\title{
Steering Control of Magnetic Helical Swimmers in Swirling Flows due to Confinement
}

\author{
Hakan O. Caldag, Serhat Yesilyurt, Senior Member, IEEE
}

\begin{abstract}
Artificial microswimmers are prospective robotic agents especially in biomedical applications. A rotating magnetic field can actuate a magnetized swimmer with a helical tail and enable propulsion. Such swimmers exhibit several modes of instability. Inside conduits, for example, hydrodynamic interactions with the boundaries lead to helical paths for pusher-mode swimmers; in this mode the helical tail pushes a rotating magnetic head. State-of-the-art in controlled navigation of microswimmers is based on aligning the swimmer orientation according to a reference path, thereby requiring both swimmer orientation and position to be known. Object-orientation is hard to track especially in in vivo scenarios which render orientationbased methods practically unfeasible. Here, we show that the kinematics for a confined swimmer can be linearized by assuming a low wobbling angle. This allows for a control law solely based on the swimmer position. The approach is demonstrated through experiments and two different numerical models: the first is based on the resistive force theory for a swimmer inside a swirling flow represented by a forced vortex and the second is a computational fluid dynamics model, which solves Stokes equations for a swimmer inside a circular channel. Helical pusher-mode trajectories are suppressed significantly for the straight path following problem. The error in real-life experiments remains comparable to those in the state-of-the-art methods.
\end{abstract}

Keywords - microswimmers, helical swimming, low Reynolds number, steering, control, stability

\section{INTRODUCTION}

Inspired by natural microorganisms such as Escherichia coli, artificial microswimmers have a great potential for targeted drug delivery and minimally invasive surgery as well as micro and nanomanipulation. Helical microswimmers are typically actuated with a rotating magnetic field, which is compatible with medical procedures [1]. The swimmers can be manufactured with dimensions ranging from millimeters to nanometers in length [2], [3]. These swimmers are either made of magnetic material or magnetized by the attachment of a permanent magnet [2]-[4].

Understanding the swimmer motion and achieving controlled navigation are crucial for enabling practical applications. Several modes of instability are reported in the literature. For example, wobbling of magnetically rotated helical swimmers is demonstrated in [2], [5], [6] and investigated theoretically in [7] based on the resistive force theory. The Mason number (Ma), which represents the ratio of viscous to magnetic torques, is critical on the wobbling; as the Mason number decreases, i.e. the magnetic torque increases, wobbling angle of a helical swimmer increases [7]. The swim-

H. O. Caldag and S. Yesilyurt are with Sabanci University, Istanbul, TURKEY (e-mail: hakancaldag@sabanciuniv.edu,

syesilyurt@sabanciuniv.edu) mers act differently under confinement due to hydrodynamic interactions. In our previous work on the motion of artificial helical swimmers in circular channels [2], we observed that the swimmers tend to follow different trajectories based on whether the tail is pushing the magnetic head (pusher-mode) or pulling (puller-mode): The pushers follow helical trajectories close to the channel boundaries while the pullers follow a straight path close to the channel centerline.

Controlled navigation of artificial swimmers has been addressed only recently. Earlier studies such as [3] and [8] show accurate in-plane control of helical swimmers in bulk fluid but they depend on open-loop algorithms due to challenges in visual feedback as stated in [9]. Xu et al. [9] are among the first to develop a closed loop control algorithm where they achieve planar path following based on the orientation error. Following this study, Oulmas et al. [10] realize the closedloop control by linearizing the swimmer dynamics through a chained formulation for tracking any 3 -dimensional path with sub-millimetric accuracy. The authors demonstrate that the closed-loop control is robust enough to overcome disturbances due to boundary effects down to $2.5 \mathrm{~mm}$ for a swimmer with a length of $14 \mathrm{~mm}$ and diameter of $1 \mathrm{~mm}$ [10]. A recent study by Leclerc et al. [11] demonstrates controlled navigation both inside and outside of a channel albeit with lower accuracy.

Here, we use steering as a control mechanism to reduce wobbling and suppress helical pusher-mode trajectories. The third Helmholtz coil pair, orthogonal to other two pairs used for the rotating magnetic field, is used to modify the normal direction of the rotation plane of the magnetic field. The tilted rotation plane of the magnetic field imposes swimmer rotation along the tilted normal which is exploited for controlled navigation. By assuming a low wobbling angle, it is possible to linearize the kinematic equations and obtain a control law based on the position and velocity of the swimmer. The novelty of this approach is that it does not require the swimmer orientation to be known, which is a significant advantage in practical applications where even observing the position of the swimmer is challenging [1]. The tracking error is comparable to those in recent studies. The performance is demonstrated in simulations and experiments.

\section{METHODOLOGY}

\section{A. Swimmer Dynamics}

Consider a swimmer inside a circular channel filled with a viscous fluid as shown in Fig. 1a. The swimmer has a cylindrical head with a length of $L_{h}$ and diameter $D_{h}$. The pitch of the helical tail is $\lambda$, tail amplitude is $B$, the number of loops is $n$, filament thickness is $d_{\text {tail }}$ and the channel diameter is $D_{c h}$. 

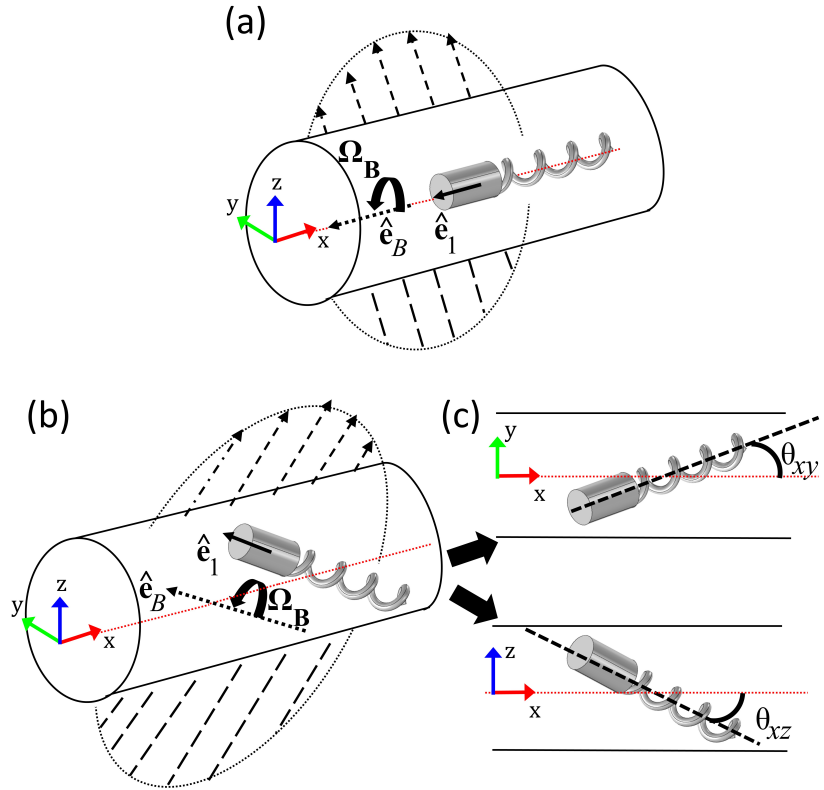

Fig. 1. (a) The geometric setup, showing the channel, the swimmer, rotating magnetic field, field normal and propulsion direction. (b) Steering of the swimmer through tilting the normal of the magnetic field. (c) Representation of angles $\theta_{x y}$ and $\theta_{x z}$.

Control of helical microswimmers is approached as a path following problem as opposed to a trajectory following problem since the swimming velocity is limited by phenomena such as step-out at high rotation frequencies and wobbling at low rotation frequencies. Here, we approach the problem as a radial path following problem and thus eliminate the need for control in the main propulsion direction which is the long axis of the channel.

The rotating magnetic field actuates the swimmer by imposing a magnetic torque on the swimmer:

$$
\boldsymbol{\tau}_{m}=\mathbf{m} \times \mathbf{Q}_{l} \mathbf{B}
$$

where $\mathbf{m}$ is the magnetization vector of the swimmer, $\mathbf{Q}_{l}$ is the rotation matrix from the lab frame (shown at Fig. 1a) to body frame of the swimmer and $\mathbf{B}$ is the total magnetic field in the lab frame. The driving magnetic field for propulsion, $\mathbf{B}_{\text {drive, }}$ is generally provided by a pair of orthogonal Helmholtz coils and expressed as:

$$
\mathbf{B}_{\text {drive }}=B_{0}\left[\begin{array}{lll}
0 & \sin (\omega t) & \cos (\omega t)
\end{array}\right]^{T}
$$

where $B_{0}$ is the amplitude of the field, $\omega$ is the rotation rate of the field and $t$ is time. Superscript "T" indicates transpose.

According to the resistive force theory (RFT) [7], viscous forces and torques $\left(\mathbf{F}_{v}\right.$ and $\left.\boldsymbol{\tau}_{v}\right)$ on the swimmer are proportional to linear and angular velocities ( $\mathbf{U}$ and $\Omega$ ) at low Reynolds number:

$$
\left[\begin{array}{l}
\mathbf{F}_{v} \\
\boldsymbol{\tau}_{v}
\end{array}\right]=\left[\begin{array}{ll}
\mathbf{R}_{L} & \mathbf{R}_{C} \\
\mathbf{R}_{C}^{\prime} & \mathbf{R}_{R}
\end{array}\right]\left[\begin{array}{l}
\mathbf{U} \\
\mathbf{\Omega}
\end{array}\right]
$$

where $\mathbf{R}_{L}, \mathbf{R}_{R}$ and $\mathbf{R}_{C}$ are called the translation, rotation and coupling matrices. The elements of the matrices depend only on the swimmer geometry in bulk swimming conditions and can be evaluated analytically for slender filaments. In the case of hydrodynamic interactions, such as near-wall swimming, the elements of the resistance matrix depend on the relative position and orientation of the swimmer with respect to solid boundaries.

\section{B. Kinematic Formulation and Control Law}

The objective of the control problem is to minimize the distance between the swimmer position $\mathbf{p}=\left[\begin{array}{lll}x & y & z\end{array}\right]^{T}$ and a reference path $\mathbf{p}_{\text {ref }}=\left[\begin{array}{lll}x & y_{\text {ref }} & z_{\text {ref }}\end{array}\right]^{T}$, meaning that no control in $\mathbf{x}$ - direction is applied. The reference path is assumed constant, e.g. $y_{r e f}=z_{r e f}=0$, to show the stability of the algorithm.

We define the state vector, $\mathbf{q}=\left[e_{y} e_{z}\right]^{\prime}$ where $e_{y}=y-y_{\text {ref }}$ and $e_{z}=z-z_{r e f}$, and its time derivatives are:

$$
\dot{\mathbf{q}}=\left[\begin{array}{l}
\dot{y}-\dot{y}_{r e f} \\
\dot{z}-\dot{z}_{r e f}
\end{array}\right]=\left[\begin{array}{l}
\dot{y} \\
\dot{z}
\end{array}\right]
$$

These velocities depend on the orientation of the swimmer, represented by the angles $\theta_{x y}$ and $\theta_{x z}$ (refer to Fig. 1c). The steering directly adjusts these angles and thus changes the propulsion direction. Orientation angles can be manipulated by the magnetic field $\mathbf{B}$. The unit normal of the rotation plane of the magnetic field, $\hat{\mathbf{e}}_{B}$, points in $\mathbf{- x}$ direction without steering, as shown at Fig. 1a. In the ideal case, the swimmer aligns its orientation in the propulsion direction, $\hat{\mathbf{e}}_{1}$, along $\hat{\mathbf{e}}_{B}$ and propels in this direction only such that the swimming velocity can be written as $\mathbf{U}=|\mathbf{U}| \hat{\mathbf{e}}_{1}$. However, helical swimmers wobble both in free and confined swimming conditions [2], [5], [6], [7]. The behavior is inherent to the swimmer geometry and it is characterized with the Mason number $(\mathrm{Ma})$, which is the ratio of hydrodynamic to magnetic torques. Due to the periodic nature of wobbling, it can be said that $\hat{\mathbf{e}}_{1}$ remains along $\hat{\mathbf{e}}_{B}$ in average. Thus, we can rewrite Eq. (4) in the following form to include the control inputs:

$$
\dot{\mathbf{q}}=\left[\begin{array}{c}
\dot{y} \\
\dot{z}
\end{array}\right]=\left[\begin{array}{l}
U \tan \left(\theta_{x y}\right) \\
U \tan \left(\theta_{x z}\right)
\end{array}\right]
$$

where $U$ is the velocity in the $\mathbf{x}$ - direction in the lab frame. Apparently, $\tan ($.$) brings non-linearity to the system, but can$ be approximated as $\tan (\theta) \sim \theta$ with error in approximation as low as $5 \%$ up to $20^{\circ}$. It was shown in Caldag et al. [2] that the orientation angles barely reach around $20^{\circ}$ when $D_{c h}=3$ $\mathrm{mm}, \lambda=1 \mathrm{~mm}$ and $n=4$ so this approximation is valid for in-channel experiments reported here. This linearization brings:

$$
\dot{\mathbf{q}}=\left[\begin{array}{c}
\dot{y} \\
\dot{z}
\end{array}\right]=\left[\begin{array}{l}
U \theta_{x y} \\
U \theta_{x z}
\end{array}\right]
$$

To drive the error to zero, error dynamics must satisfy:

$$
\dot{\mathbf{q}}+\left[\begin{array}{ll}
K_{y} & K_{z}
\end{array}\right] \mathbf{q}=0
$$

for positive gains, $K_{\{y, z\}}>0$. Then according to (6), we have: 


$$
U \theta_{x\{y, z\}}+K_{\{y, z\}} e_{\{y, z\}}=0
$$

Thus, the control inputs are:

$$
\theta_{x\{y, z\}}=\frac{-K_{\{y, z\}} e_{\{y, z\}}}{U}
$$

Steering is realized through an additional magnetic field,

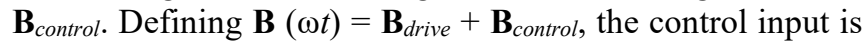
as following:

$$
\mathbf{B}_{\text {control }}=[\alpha \cos (\omega t)+\beta \sin (\omega t) \quad 0 \quad 0]^{T}
$$

with coefficients $\alpha$ and $\beta$. $\hat{\mathbf{e}}_{B}$ for the tilted magnetic field can be evaluated from the cross product of a pair of orthogonal magnetic field vectors:

$$
\hat{\mathbf{e}}_{B}=\frac{\mathbf{B}(0) \times \mathbf{B}(\pi / 2)}{\|\mathbf{B}(0) \times \mathbf{B}(\pi / 2)\|}=\frac{\left[\begin{array}{lll}
1 & -\beta & -\alpha
\end{array}\right]}{\sqrt{1+\alpha^{2}+\beta^{2}}}
$$

If $\beta=0$ and $\alpha \neq 0, \hat{\mathbf{e}}_{B}$ is tilted in $\mathbf{z}$ - direction only, as demonstrated at Fig. 1b. For a non-zero $\alpha, \hat{\mathbf{e}}_{B}$ will be tilted in the $\mathbf{y}$-direction as well. The magnitudes of the coefficients will adjust the scaling. This mechanism can be combined with a simple feedback controller to move the swimmer towards $\mathbf{p}_{\text {ref }}$ :

$$
\mathbf{B}_{\text {control }}=\mathbf{K}_{p}\left(\mathbf{p}-\mathbf{p}_{\text {ref }}\right)
$$

where $\mathbf{K}_{p}$ is a $3 \times 3$ matrix that contains the proportional gains and time-dependent parts of the magnetic field. In this configuration, matrix $\mathbf{K}_{p}$ will have the following form:

$$
\mathbf{K}_{p}=\left[\begin{array}{ccc}
0 & K_{p, y} \cos (\omega t) & K_{p, z} \sin (\omega t) \\
0 & 0 & 0 \\
0 & 0 & 0
\end{array}\right]
$$

Therefore, the coefficients $\alpha$ and $\beta$ in Eq. (10) can be expressed in terms of the position error and the gains, $K_{p, y}$ and $K_{p, z}$ as follows:

$$
\alpha=K_{p, y} e_{y}, \quad \beta=-K_{p, z} e_{z}
$$

The relation between the tilting and the coefficients is:

$$
\theta_{x y}=\arctan (-\beta), \quad \theta_{x z}=\arctan (-\alpha)
$$

Rewriting Eq. (9), we obtain:

$$
\theta_{x y}=\arctan \left(-K_{p, y} e_{y}\right)=\frac{K_{y} e_{y}}{U}
$$

Based on the $\tan (\theta) \sim \theta$ assumption, the relation between $K_{p,\{y, z\}}$ and $K_{\{y, z\}}$ come out as:

$$
\begin{aligned}
& K_{p, y}=\frac{K_{y}}{U} \\
& K_{p, z}=\frac{K_{z}}{U}
\end{aligned}
$$

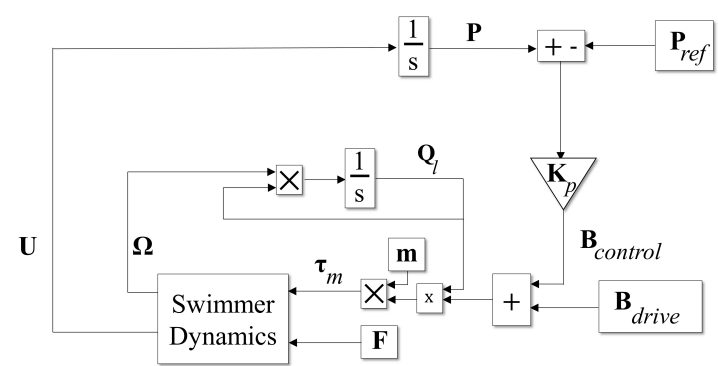

Fig. 2. Block diagram of swimmer kinematics and control.

Overall dynamics of the swimmer and control mechanism are drawn as a block diagram in Fig. 2. The swimmer is continually tilted towards the reference path with no knowledge of swimmer orientation. This is advantageous especially considering the potential in vivo applications where even obtaining accurate swimmer position information is challenging.

For stability analysis, we define a Lyapunov function, $V$ as:

$$
V=\frac{1}{2}\left(e_{y}^{2}+e_{z}^{2}\right)
$$

It is obvious that $V=0$ for $\left\{e_{y}, e_{z}\right\}=\{0,0\}$ and $V>0$ for all $\left\{e_{y}, e_{z}\right\}-\{0,0\}$. If we take the time derivative, assuming that $y_{\text {ref }}$ and $z_{\text {ref }}$ are constant in time:

$$
\dot{V}=\left(e_{y}\right)(\dot{y})+\left(e_{z}\right)(\dot{z})
$$

Here, the velocities $\dot{y}$ and $\dot{z}$ are the control inputs set via $\mathbf{B}_{\text {control. }}$ In order to satisfy $\dot{V}<0$ for all $\left\{e_{y}, e_{z}\right\}-\{0,0\}, \dot{y}$ and $\dot{z}$ should have opposite signs with $e_{y}$ and $e_{z}$, respectively. In other words, the swimmer should be tilted upward when it is below the reference path and vice versa. The definitions of $\alpha$ and $\beta$ in Eq. (14) alongside their effects on $\hat{\mathbf{e}}_{1}$ show that this is always the case. Since all Lyapunov stability conditions are satisfied, $\left\{e_{y}, e_{z}\right\}=\{0,0\}$ is a stable equilibrium point. Even though this stability analysis is not complete for time-varying $\mathbf{p}_{\text {ref, }}$, the results in Section III show that time-varying paths can be followed as well.

Even though the proportional control is sufficient in most of the cases tested here (refer to Section III), integral and derivative control are incorporated in the experimental setup to see whether there would be any improvement in the performance. Additional terms are introduced to Eq. (12) as the following:

$$
\begin{aligned}
& \mathbf{B}_{\text {control }}=\mathbf{K}_{p}\left(\mathbf{p}-\mathbf{p}_{r e f}\right)+\mathbf{K}_{i} \int_{0}^{t}\left(\mathbf{p}-\mathbf{p}_{r e f}\right) d t \\
& +\mathbf{K}_{d} \frac{d\left(\mathbf{p}-\mathbf{p}_{r e f}\right)}{d t}
\end{aligned}
$$

Integral evaluation is carried out by trapezoidal method while the derivative of the error is approximated with a Savitzky-Golay filter that uses 10 previous datapoints. Matrices $\mathbf{K}_{i}$ and $\mathbf{K}_{d}$ have a form similar to $\mathbf{K}_{p}$ in Eq. (13) with the gains $K_{i,\{y, z\}}$ and $K_{d,\{y, z\}}$. 


\section{Numerical Models}

Steering control is validated with two different numerical models. The first one is based on the resistive force theory with the assumption that the swimmer has a thin helical tail as considered in [7]. Here, we use simplified expressions that fully match the ones provided in [7]. By employing Eq. (3), instantaneous linear and angular velocities are obtained at a given time using the magnetic torque, $\boldsymbol{\tau}_{m}$ in Eq. (1) and the specified magnetic field $\mathbf{B}$ (other forces and torques, such as gravity and contact, are neglected). Velocities are used to compute the complete trajectories with kinematic equations:

$$
\begin{gathered}
\frac{d \mathbf{p}}{d t}=\mathbf{U} \\
\frac{d \mathbf{e}_{i}}{d t}=\mathbf{\Omega} \times \hat{\mathbf{e}}_{i}
\end{gathered}
$$

where $\hat{\mathbf{e}}_{i}$ for $i=1,2,3$ correspond to the unit vectors of the body coordinate frame.

As the swimmer rotates inside the channel, it generates a swirling flow in the channel. We introduce a forced vortex through a swirl flow with angular velocity $\Gamma$ to mimic the swimming inside a circular channel. The tangential velocity is decomposed into:

$$
\begin{aligned}
& U_{f, y}=-\Gamma z \\
& U_{f, z}=\Gamma y
\end{aligned}
$$

Drag forces due to these additional velocities are added in (3). Note that these velocities are in the lab frame, thus the additional drag forces should be evaluated locally and summed up. In order to do that, the velocities have to be expressed in the local coordinate frame. Defining $s$ as the curvilinear coordinate index that represents the tail, $\mathbf{U}_{f}$ in the body frame at position $s$ is:

$$
\mathbf{U}_{f}^{b}(s)=\mathbf{Q}_{l} \mathbf{W}\left(\mathbf{Q}_{l}^{T} \mathbf{x}_{l}+\mathbf{x}\right)
$$

where $\mathbf{x}_{l}$ represents the coordinates of a local position on the helical tail in body coordinates as a function of $s$ and $\mathbf{W}$ represents the multiplications in Eq. (24) with $\mathbf{W}(2,3)=-\Gamma$, $\mathbf{W}(3,2)=\Gamma$ and the rest are zeroes. The normal and tangential components of the differential force due to swirl are then evaluated as:

$$
\begin{aligned}
\mathbf{d F}_{s w, \mathbf{t}} & =c_{t}\left(\mathbf{U}_{\mathbf{f}}^{\mathbf{b}} \cdot \hat{\mathbf{t}}\right) \hat{\mathbf{t}} \\
\mathbf{d F}_{s w, \mathbf{n}} & =c_{n}\left(\mathbf{U}_{\mathbf{f}}^{\mathbf{b}}-\mathbf{U}_{\mathbf{f}}^{\mathbf{b}} \cdot \hat{\mathbf{t}}\right) \hat{\mathbf{t}}
\end{aligned}
$$

where $\hat{\mathbf{t}}$ is the unit vector in tangential direction on the helical tail, the superscript " $b$ " indicates the body frame of the swimmer, $c_{t}$ and $c_{n}$ are the tangential and normal resistive coefficients, respectively:

$$
c_{t}=\frac{2 \pi \mu}{\log \left(\frac{2 \lambda}{d_{\text {tail }}}\right)-\frac{1}{2}}, \quad c_{n}=2 c_{t}
$$

where $\mu$ is the fluid viscosity. The elements of the resistance matrices in Eq. (3) were derived in a non-dimensional fash- ion in [7]. Here, we use the simplified versions of those expressions which output exactly the same results with those in [7]. Defining:

$$
\begin{gathered}
k_{w}=\frac{2 \pi}{\lambda}, \quad C=\frac{c_{t} n}{\sqrt{1+k_{w}^{2} B^{2}}}, \quad a=\frac{1}{\sqrt{1+k_{w}^{2} B^{2}}} \\
A_{1}=-\frac{C}{\lambda}, \quad A_{2}=3 \pi^{2} B^{2}, A_{3}=A_{2}+\lambda^{2} \\
\mathrm{~A}_{4}=k_{w}^{2} B^{4}+\frac{2}{3} A_{2} n^{2}-\frac{1}{4} B^{2}+\frac{2}{3} \lambda^{2} n^{2} \\
\mathrm{~A}_{5}=k_{w}^{2} B^{4}+\frac{2}{3} A_{2} n^{2}-\frac{5}{4} B^{2}+\frac{2}{3} \lambda^{2} n^{2}
\end{gathered}
$$

The resistance matrices come out as following:

$$
\begin{gathered}
\mathbf{R}_{L}=A_{1}\left[\begin{array}{ccc}
2 A_{3} & 0 & 0 \\
0 & 2 A_{3} & 0 \\
0 & 0 & \frac{8}{3} A_{2}+\lambda^{2}
\end{array}\right] \\
\mathbf{R}_{C}=A_{1}\left[\begin{array}{ccc}
\frac{\lambda A_{2}}{2 \pi} & n \lambda A_{3} & 0 \\
-n \lambda A_{3} & \frac{\lambda A_{2}}{6 \pi} & 0 \\
0 & -\lambda^{2} B & -2 \pi \lambda B^{2}
\end{array}\right]
\end{gathered}
$$

$\mathbf{R}_{R}=A_{1} \lambda^{2}\left[\begin{array}{ccc}A_{4} & \frac{n A_{2}}{6} & 0 \\ \frac{n A_{2}}{6} & A_{5} & \frac{B}{\pi \lambda}\left(\frac{2}{3} A_{2}+\lambda^{2}\right) \\ 0 & \frac{B}{\pi \lambda}\left(\frac{2}{3} A_{2}+\lambda^{2}\right) & \frac{2 B}{\lambda}\left(\frac{2}{3} A_{2}+\lambda^{2}\right)\end{array}\right]$

Note that the geometric parameters in these expressions were defined in Section II-A.

Apart from this numerical model, the control algorithm is also tested with a CFD-based kinematic model that incorporates snapshot solutions to the Stokes equations to solve for instantaneous swimmer velocities which are then integrated to evaluate the swimmer position at the next time step by using Eqs. (22)-(23). The geometric setup is as shown in Fig. 1a as in-channel swimming can be simulated exactly with the help of complete solutions to Stokes equations. As the magnetic field can be changed at each instant simulated, the response time of the control algorithm is as low as $\Delta t=$ 0.0016 seconds for the simulations reported here. Verification of this model and finer details are out of the scope of this work and the reader is referred to [12] for further details.

\section{Experiment Setup}

The experiment setup consists of 3 pairs of orthogonally placed Helmholtz coils, as shown in Fig. 3. The helical microswimmer is produced with $3 \mathrm{D}$ printing and it is magnetized by attaching a radially magnetized cylindrical magnet to its head. The swimmer is placed inside a glycerol-filled 


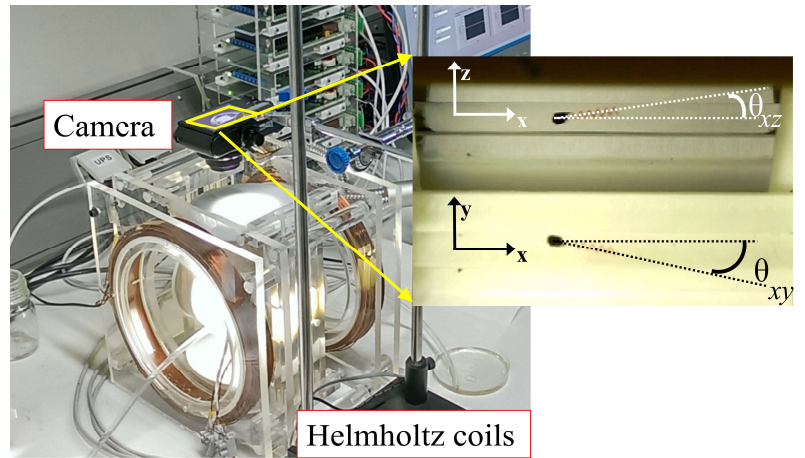

Fig. 3. Experimental setup showing the Helmholtz coils, digital camera and computer. An image captured from experiment recordings; coordinate axes and angles $\theta_{x y}$ and $\theta_{x z}$ are shown at the right-hand side.

cylindrical channel for low Reynolds number swimming. Geometric properties of the swimmer are listed in Table I. Swimmer position is extracted from the images recorded in real-time with a digital camera placed above the setup. A mirror is placed next to the channel with 45-degree inclination and allows the extraction of $3 \mathrm{D}$ position information using Matlab's Image Processing Toolbox (see Fig. 3). Further details of the experiment setup can be found in [2].

\section{RESULTS}

Stabilizing the pusher-mode swimming in channels is a challenging and important task as the swimmers follow helical trajectories and crash into the walls occasionally. In this study, steering control is implemented to suppress helical trajectories in the pusher-mode. The algorithm is first tested in the RFT-based model presented in Section II. The swimmer geometry is as defined in [7] with $n=3$. Initial position is set to $\mathbf{p}_{0}=\left[\begin{array}{lll}0 & 1 & 1\end{array}\right]^{T}$ and $\mathbf{p}_{r e f}=\left[\begin{array}{lll}x & 0 & 0\end{array}\right]^{T}$. In accordance with the calculations from our previous simulations for a confined swimmer, the Mason number, $\mathrm{Ma}$, is set to 1 [12]; the Mason number is defined as:

$$
\mathrm{Ma}=\frac{\mu \omega \lambda^{3}}{|\mathbf{m}| B_{0}}
$$

Swirling flow is the main cause of the helical trajectory of the swimmer. The strength of the forced vortex, $\Gamma$, is taken as $\pi / 10$ such that it is significantly lower than the nondimensional rotation rate of the magnetic field, $2 \pi$, similar to the observed swirl rate in experiments. Trajectories under different control gains are displayed in Fig. 4. Control algorithm significantly suppresses the helical path as the radius of the trajectory falls from around $5 \mathrm{~mm}$ to $0.27 \mathrm{~mm}$ and to $0.05 \mathrm{~mm}$ for the highest gain value tested. Note that this improvement increases propulsion velocity as well, from $0.08 \mathrm{~mm} / \mathrm{s}$ without control to $0.35 \mathrm{~mm} / \mathrm{s}$ and to $0.36 \mathrm{~mm} / \mathrm{s}$

Table I. Geometric parameters of the swimmer used in experiments.

\begin{tabular}{|c|c|}
\hline Geometric Parameter & Value \\
\hline$D_{h}[\mathrm{~mm}]$ & 0.8 \\
\hline$L_{h}[\mathrm{~mm}]$ & 1.5 \\
\hline$L[\mathrm{~mm}]$ & 4 \\
\hline$\lambda[\mathrm{mm}]$ & 1 \\
\hline$d_{\text {tail }}[\mathrm{mm}]$ & 0.2 \\
\hline
\end{tabular}
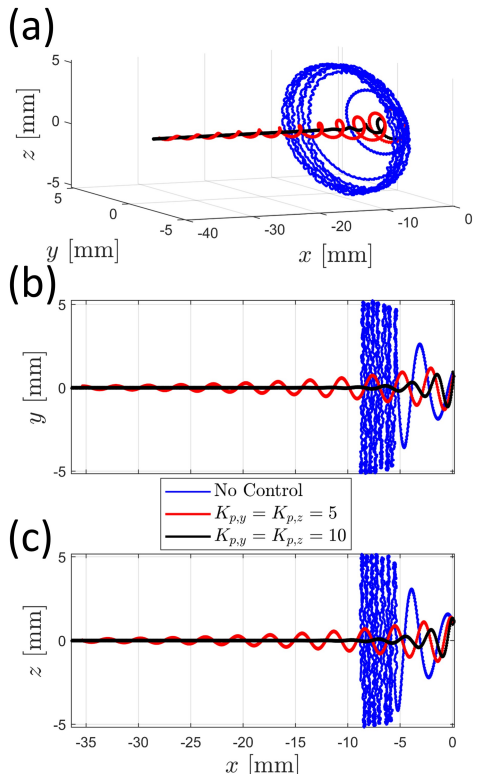

Fig. 4. Swimmer trajectories in the numerical model with and without control. (a) shows the 3D trajectories while (b) shows $y$ vs. $x$ and (c) shows $z$ vs. $x$.

with the control in the loop. The control algorithm performs well in the kinematic model too where the complete Stokes equations are solved. Here, $D_{c h}=3 \mathrm{~mm}$ and control gains are set to unity. Fig. 5a shows that the trajectory radius decreases from around $0.71 \mathrm{~mm}$ to $0.05 \mathrm{~mm}$, which is the threshold value for the algorithm to stop steering. As the change in the trajectory radius is much lower compared to the unbounded swimmer in the numerical model, the improvement in the propulsion velocity is negligible here. It is also possible to make the swimmer follow time-varying paths with this algorithm as shown at Figs. 5b-c where $\mathbf{p}_{r e f}=\left[\begin{array}{lll}x & 0 & 0.2 \sin (1.5 t)\end{array}\right]^{\prime}$. This would hint at potential $3 \mathrm{D}$ path following in complex environments.

Next, we test the control algorithm experimentally. $\mathbf{p}_{\text {ref }}=\left[\begin{array}{lll}x & 0 & 0\end{array}\right]^{T}$ for all cases with an error threshold of \pm 0.1 $\mathrm{mm}$ (this is larger compared to the simulations in order to account for the error in position estimation of the swimmer) and $K_{p, y}=K_{p, z}=1$ again. This value is concluded by testing different gain values (not shown). Rotation rate of the magnetic field is set to $10 \mathrm{~Hz}, B_{0} \sim 5 \mathrm{mT}$ and $|\mathbf{m}| \sim 6 \times 10^{-4}$ A.m ${ }^{2}$. This configuration prevents step-out [5] while providing a strong propulsion to the swimmer. Note that gravitational effects are negligible at these rotation rates as the swirling flow lifts the swimmer, hence, there is no additional compensation for gravity. Figs. 6a-b show the non-dimensional radial position $(\beta)$ of the swimmer, defined as:

$$
\beta=\frac{r}{R_{c h}-R_{s w}}
$$

where $r$ is the radial position of the swimmer on $\mathrm{y}-\mathrm{z}$ plane, $R_{c h}=D_{c h} / 2$ and $R_{s w}$ is the radius of the swimmer head. While the helical path is suppressed significantly in both cases, the swimmer reaches to the reference path within the threshold for $D_{c h}=3 \mathrm{~mm}$ but fails in the 5.6- $\mathrm{mm}$ channel within the duration of the experiment. The length of the experiment setup alongside the channel axis is too short to 
(a)
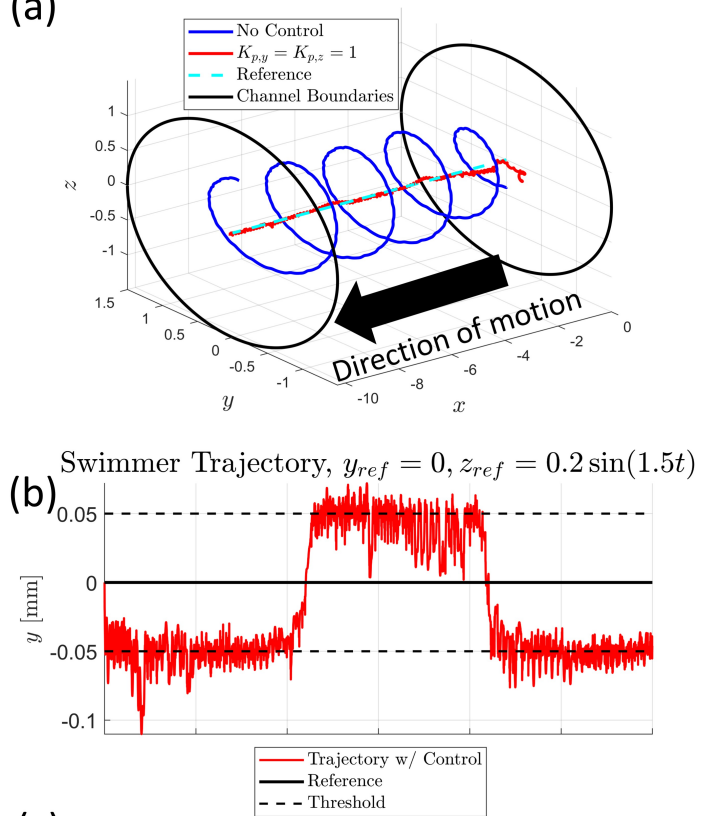

(c)

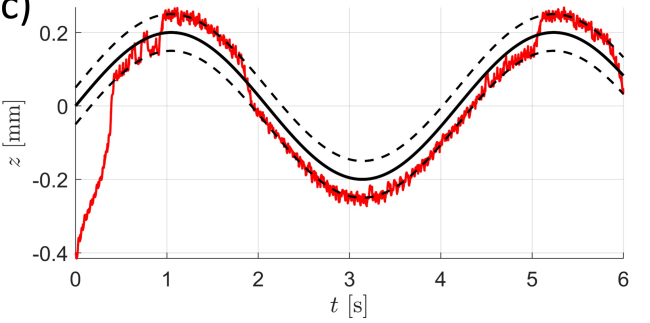

Fig. 5. (a) 3D swimmer trajectories in the pusher-mode from the kinematic model with and without control. (b) $\mathbf{y}$ - coordinates and (c) zcoordinates of the swimmer for $\mathbf{p}_{\text {ref }}=\left[\begin{array}{lll}x & 0 & 0.2 \sin (1.5 \mathrm{t})\end{array}\right]^{T}$.

show fully converged trajectories inside the wider channel but there is a significant decrease in $\beta$ compared to the case without control. Noting that the same control gains are applied in both cases, it is natural that the algorithm performs worse for a wider channel where Ma and swirl rates are lower. While the swirling flow causes helical trajectories, it is beneficial in achieving quicker convergence to the reference path. The control input could not be increased further due to hardware limitations. There is also the issue of delayed response due to hardware and image processing. Here, the control input is updated at each rotation of the swimmer, corresponding to a response time of $0.1 \mathrm{~s}$. The algorithm should perform better if these problems are overcome, as the numerical results indicate.

We conduct additional experiments for $D_{c h}=3 \mathrm{~mm}$. P, PD, PI and PID control are tested as defined in Eq. (23). The change of $\beta$ under these scenarios are shown in Fig. $6 c$ (the trajectory with PD control is omitted as it is very similar to the trajectory with PID control). PI control increases the oscillations in the system while P, PD and PID control result in similar paths under optimal gain values. The reason for similar performance can be attributed to the limitations in suppression of the helical paths. $\mathrm{P}$ control appears to be sufficient in suppression to a degree while these additional inputs cannot contribute any further. Wobbling cannot be suppressed completely either, as shown in the simulation results in Figs. 4 and 5. As Ma number is finite, some
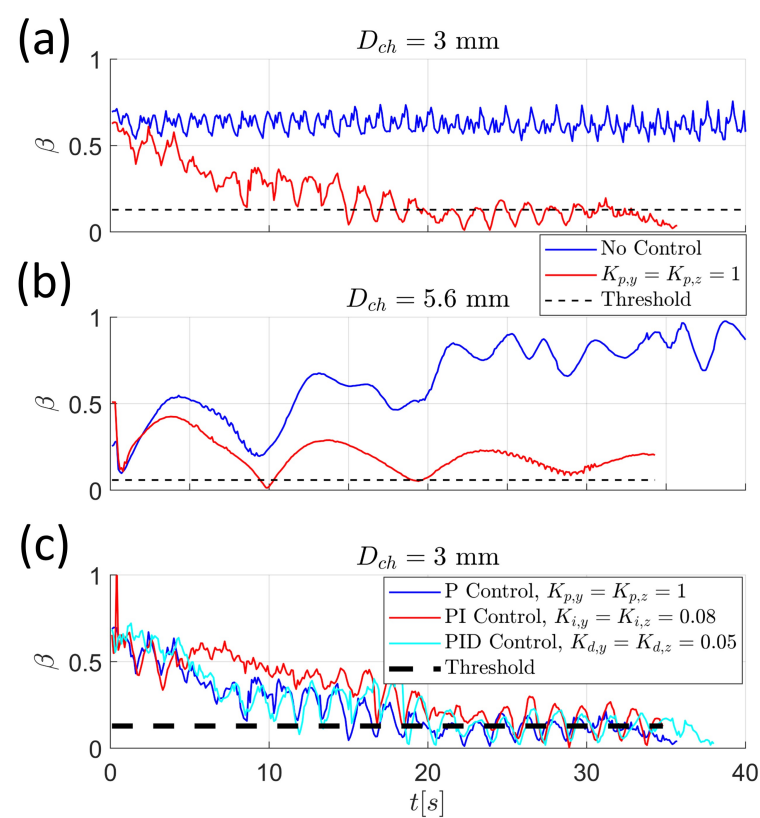

Fig. 6. Non-dimensional trajectory radii with and without control in the channels where (a) $D_{c h}=3 \mathrm{~mm}$, (b) $D_{c h}=5.6 \mathrm{~mm}$. (c) shows the performance of different control strategies under $D_{c h}=3 \mathrm{~mm}$. Note that $K_{i,\{y, z\}}$ for PID control are the same as in PI control.

wobbling will remain even if Ma is very high. Higher integral or derivative gains quickly destabilize the system because of the oscillatory nature of the trajectories. Higher Pgain could improve the control performance but the increase in magnetic torque may trigger more wobbling, so it cannot be increased indefinitely.

\section{CONCLUSION}

Reports on the locomotion of natural and artificial microswimmers show several modes of instability. The helical swimmers exhibit wobbling in bulk that is inherent to their geometry and it cannot be overcome with geometric modifications and control algorithms, especially at low Mason numbers corresponding to high magnetic fields. Moreover, due to hydrodynamic interactions, helical trajectories are observed in confined swimming in the case of pusher-mode swimming. Here, a proportional control algorithm is demonstrated on the suppression of helical pusher-mode trajectories. The method is based on steering of the swimmer by tilting the rotation plane of the driving magnetic field. For misalignment angles less than $20^{\circ}$, there is no need to extract the swimmer orientation. At high Ma numbers, the swimmer can be controlled effectively with this approach. As the confinement increases the viscous effects, the proposed method is particularly useful in such scenarios.

The extension of the framework to 3D path following and reduction in the response time of the control algorithm in experiments constitute the future work. This approach is expected to be adoptable for practical applications.

\section{REFERENCES}

S. Martel, "Microrobotics in the vascular network: present status and next challenges," J. Micro-Bio Robot., vol. 8, no. 1, pp. 41-52, 2013. H. O. Caldag, A. Acemoglu, and S. Yesilyurt, 
"Experimental characterization of helical swimming trajectories in circular channels," Microfluid. Nanofluidics, vol. 21, no. 8, pp. 1-13, 2017.

[3] A. Ghosh and P. Fischer, "Controlled Propulsion of Artificial Magnetic Nanostructured Propellers,"

Nano Lett., vol. 9, no. 6, pp. 2243-2245, 2009.

[4] C. Peters, O. Ergeneman, B. J. Nelson, and C.

Hierold, "Superparamagnetic swimming microrobots with adjusted magnetic anisotropy," Proc. IEEE Int. Conf. Micro Electro Mech. Syst., pp. 564-567, 2013.

[5] L. Zhang, J. J. Abbott, L. Dong, B. E. Kratochvil, D. Bell, and B. J. Nelson, "Artificial bacterial flagella: Fabrication and magnetic control," Appl. Phys. Lett., vol. 94, no. 6, 2009.

[6] K. E. Peyer, L. Zhang, B. E. Kratochvil, and B. J. Nelson, "Non-ideal swimming of artificial bacterial flagella near a surface," Proc. - IEEE Int. Conf.

Robot. Autom., pp. 96-101, 2010.

[7] Y. Man and E. Lauga, "The wobbling-to-swimming transition of rotated helices," Phys. Fluids, vol. 25, no. 7, pp. 1-16, 2013.

[8] S. Tottori, L. Zhang, F. Qiu, K. K. Krawczyk, A. Franco-Obregõn, and B. J. Nelson, "Magnetic helical micromachines: Fabrication, controlled swimming, and cargo transport," Adv. Mater., vol. 24, no. 6, pp. 811-816, 2012.

[9] T. Xu, G. Hwang, N. Andreff, and S. Régnier, "Planar path following of 3-D steering scaled-up helical microswimmers," IEEE Trans. Robot., vol. 31, no. 1, pp. 117-127, 2015.

[10] A. Oulmas, N. Andreff, and S. Régnier, "3D closedloop swimming at low Reynolds numbers," Int. J. Rob. Res., vol. 37, no. 11, pp. 1359-1375, 2018.

[11] J. Leclerc, H. Zhao, and A. T. Becker, "3D Control of Rotating Millimeter-Scale Swimmers Through Obstacles," IEEE Int. Conf. Robot. Autom., pp. 8890-8896, 2019.

[12] H. O. Caldag and S. Yesilyurt, "Trajectories of magnetically-actuated helical swimmers in cylindrical channels at low Reynolds numbers," $J$. Fluids Struct., vol. 90, pp. 164-176, 2019. 